Forschungszentrum KaRLsRUhe

Technik und Umwelt

\author{
Wissenschaftliche Berichte \\ FZKA 6105
}

\title{
Core Structure of Extensive Air Showers at Primary Energies around the Knee by a Multifractal Moments Analysis
}

\author{
A. Haungs, J. Kempa ${ }^{1}$, H. Rebel,
} W.D. Apel, A.F. Badea 2 , K. Bekk, E. Bollmann, H. Bozdog', I.M. Brancus ${ }^{2}$, M. Brendle ${ }^{3}$, A. Chilingarian ${ }^{4}$, K. Daumiller ${ }^{5}$, P. Doll, J. Engler, H.J. Gils, R. Glasstetter, R. Haeusler, W. Hafemann, D. Heck, J. Hörandel, K.H. Kampert, H. Keim, H.O. Klages, J. Knapp ${ }^{5}$, H.J. Mathes, H.J. Mayer, D. Mühlenberg, J. Oehlschläger, M. Petcu ${ }^{2}$, M. Risse, M. Roth, G. Schatz, F.K. Schmidt ${ }^{5}$, T. Thouw, B. Vulpescu ${ }^{2}$, G.J. Wagner ${ }^{3}$, J.H. Weber, J. Wentz, T. Wibig ${ }^{1}$, T. Wiegert, D. Wochele, J. Wochele, J. Zabierowski ${ }^{6}$, S. Zagromski

Institut für Kernphysik

${ }^{1}$ Department of Experimental Physics, Univ. Lodz, Lodz, Poland ${ }^{2}$ Institute of Physics and Nuclear Engineering, Bucharest, Romania ${ }^{3}$ Physikalisches Institut, Univ. Tübingen, Germany ${ }^{4}$ Cosmic Ray Division, Yerevan Physics Institute, Yerevan, Armenia ${ }^{5}$ Institut für Experimentelle Kernphysik, Univ. Karlsruhe, Germany ${ }^{6}$ Soltan Institute for Nuclear Studies, Lodz, Poland Forschungszentrum Karlsruhe GmbH, Karlsruhe 



\begin{abstract}
A system of large-area position-sensitive multiwire proportional chambers (MWPC), installed below the hadron calorimeter of the central detector of the KASCADE (KArlsruhe Shower Core and Array DEtector) experiment is able to observe the density distributions of high-energy muons and of the high-energy tails of the hadron spectrum in the core of extensive air showers (EAS). Applying a classification by multifractal moments of the observed patterns allows to explore the information on mass composition of cosmic rays in the studied energy region. Additionally correlating with the shower size and the number of reconstructed muons in the core region, an artificial neural net analysis leads to probability distributions for associating single showers to particular primary masses. The classification can serve as basis of a more detailed shower specification considering further measured shower parameters.
\end{abstract}

\title{
Strukturen des Kerns von ausgedehnten Luftschauern im Bereich des Knies aus einer Analyse multifraktaler Momente
}

Ein System von großflächigen ortsaufösenden Vieldraht-Proportionalkammern (MWPC), die unterhalb des Hadronkalorimeters $\left(1000 \mathrm{~g} / \mathrm{cm}^{2} \mathrm{Fe}\right)$ des Zentraldetektors installiert sind, erlaubt die Messung von Dichteverteilungen hochenergetischer Myonen und hochenergetischer, den Eisenabsorber durchdringenden Hadronen ausgedehnter Luftschauer (EAS). Mit Hilfe einer Klassifizierung der Verteilungsmuster nach multifraktalen Momenten können Informationen über die Masse des Primärteilchens gewonnen werden. Korreliert mit der Schauergröße und der Zahl der im Schauerzentrum rekonstruierten Myonen führt ein neuronales Netz zu Wahrscheinlichkeitsverteilungen in der primären Masse, die Ausgangspunkt einer detaillierteren Spezifikation der beobachteten EAS sind. 



\section{Introduction}

Quae visa, vera; quae non, veriora

(What you see is true, what you not see is even more true)

J.Masters, Coromandel

The energy spectrum of primary cosmic rays follows power laws $\propto E^{-\alpha}$ in the range from $10^{11} \mathrm{eV}$ up to $10^{19} \mathrm{eV}$. Near $1 \mathrm{PeV}$ the exponent $\alpha$ shows a distinct change from ca. 2.7 to ca. 3.1 [1]. The origin of this kink, the so-called "knee" is a matter of current discussion. An experimental demonstration of a changing chemical composition of the primary particles in this energy region would considerably constrain the speculations and theoretical conjectures for explaining the sources, the acceleration and the transport mechanisms of high-energy cosmic rays.

$\mathrm{Up}$ to $10^{14} \mathrm{eV}$ the elemental spectra can be inferred from direct measurements with satellite or balloon-borne experiments. They exhibit only a small variation of the chemical composition [2]. The steeply falling intensity of the primary particle flux limits the extension of direct measurements to higher energies. The standard technique above $10^{14} \mathrm{eV}$ is the observation and analysis of "extensive air showers" (EAS), induced by the primary particles when penetrating into the atmosphere and producing a huge number of secondaries by cascading processes of successive interactions with atmospheric nuclei. The main observable particle components of such a shower are the electron-photon, the muonic, and the hadronic components. Due to different energies per nucleon, different interaction lengths and multiplicities of secondary particle production primary particles of the same total energy, but of different masses develop differently in the atmosphere. As a result differences in intensities, energies and lateral distributions of the secondaries reaching the observation level are found. For mass discrimination the most powerful observable, measured in earthbound EAS experiments is the correlation of the total intensity of the electron-photon component with the total or partial number of muons of the shower.

Measurements of such kind are the task of the field array of the KASCADE [3] experiment (setup at sea level), a sampling detector system of an array of 252 detector stations to determine angle-of-incidence, core location, the electron and muon content of individual showers. The coverage of sensitive area is ca. two percent and better than in comparable experiments. With the reduction of instrumental uncertainties the influence of intrinsic fluctuations of the shower development, obscuring the mass discriminating signals, remains as one of the dominant problems. It is the concept of the KASCADE approach to relieve this kind of problems by measuring a larger number of mass sensitive parameters for each single shower which can be correlated in an analysis of multivariate distributions [3]. In addition the uncertainties arising from the insufficiently known hadronic interaction at high energies, entering in the Monte Carlo simulation analysis could be minimized. This is particularly the case by studies of the hadronic EAS core. For such investigations in the center of the field array of KASCADE a central detector is set up, basically consisting of an iron sampling calorimeter to study the lateral and energy distributions of the hadron component. The third active layer of the calorimeter is a system of fast scintillators, used for measurements of muon arrival time distributions and for triggering the ad- 
ditional detector devices, in particular a setup of multiwire proportional chambers (MWPC) in the basement of the central detector. Exploiting the relatively good spatial resolution the MWPC are used for identification of muons with energies larger than $2 \mathrm{GeV}$ and for studies of the lateral distribution of particles, which penetrate through the calorimeter absorber. They are dominanted by high-energy muons, but also by the high-energy tails of the spectrum of "punch-through" hadrons of the shower core. The MWPC are arranged in double layers, thus with a telescope effect improving the reconstruction quality of the particle hits and informing about the muon direction. The pattern of the spatial distribution of the tracked muons and all reconstructed secondaries in the MWPC carry mass-sensitive information. The sensitivity arises from the differences of the EAS development in the multiplicity distributions, transverse-momentum distributions, and energy spectra of the secondary particle production.

Detailed simulation studies [4] have shown, that the mass separation quality of the observed particle density distributions for single showers, measurable by the MWPC system, is rather promising for showers of the same shower size, at least. The actual analysis is based on the method of multifractal moments. Since the theoretical introduction by Mandelbrot 1967 [5] multifractal moments analyses are used for consideration of various different problems of physics, like heavy ion interactions [6], large scale structures of matter in the Universe [7] and also in cosmic ray studies by analyses of the core of the $e / \gamma$-component of EAS [8].

In this paper we apply the method to the analysis of single showers measured by the KASCADE detector system and explore the information on the primary mass, hidden in the structure of the shower core of an EAS. A neural network classification allows to group the data in samples of enhanced light and heavy induced showers. In addition to an estimate of the variation of the ratio of light nuclei to heavy nuclei in the primary energy spectrum, a comparison of this information with independently measured observables reveal the features of cosmic rays around the knee and the tendencies of the primary mass composition.

\section{The Central Detector Facilities of KASCADE}

In the center of the KASCADE array which provides the basic EAS information on shower direction and core location there is the KASCADE central detector (Fig.1), composed of various different detector components. Basically the setup is an iron sampling calorimeter [9] for identification and energy measurement of hadrons, especially in the shower core. The active detectors, distributed in 8 layers and measuring the energy loss of traversing hadrons, are room temperature liquid (tetramethylsilane or tetramethylpentane) ionisation chambers. From the recorded signals of the energy deposits hadrons can be reconstructed with energies from $20 \mathrm{GeV}$ up to some $10 \mathrm{TeV}$. In the basement of the setup, below ca. $3800 \mathrm{t}$ of iron and concrete, there is an installation of position-sensitive multiwire proportional chambers (MWPC) for identification of muons [10] with an energy larger than $2 \mathrm{GeV}$. Due to the relative good spatial resolution (ca. $8 \mathrm{~mm}$ ) the MWPC, arranged in sixteen double-stacks (Fig.2) and with a sensitive area of $122 \mathrm{~m}^{2}$ covering more than 60 percent of a central area with $R<8 \mathrm{~m}$, allow detailed measurements of the lateral distribution 
and intensity of particles, penetrating the absorber above, mainly muons, but in the shower core also the "punch-through" of high-energy hadrons. Such measurements are the experimental basis of the present investigation.

Each muon chamber consists of three layers of crossed wires and stripes (Fig.3) in a $1.6 \mathrm{~cm}$ thick argon-methane volume which allow a reconstruction of the crossing

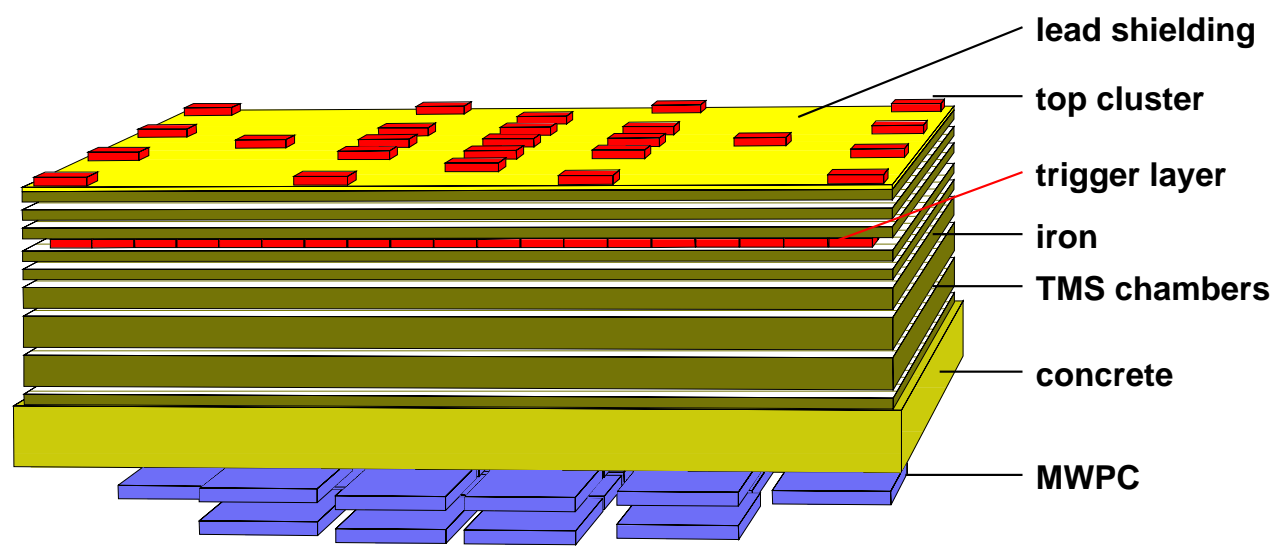

Figure 1: Schematic layout of the central detector system of KASCADE $(16 m \times 20 m \times 5 m)$.

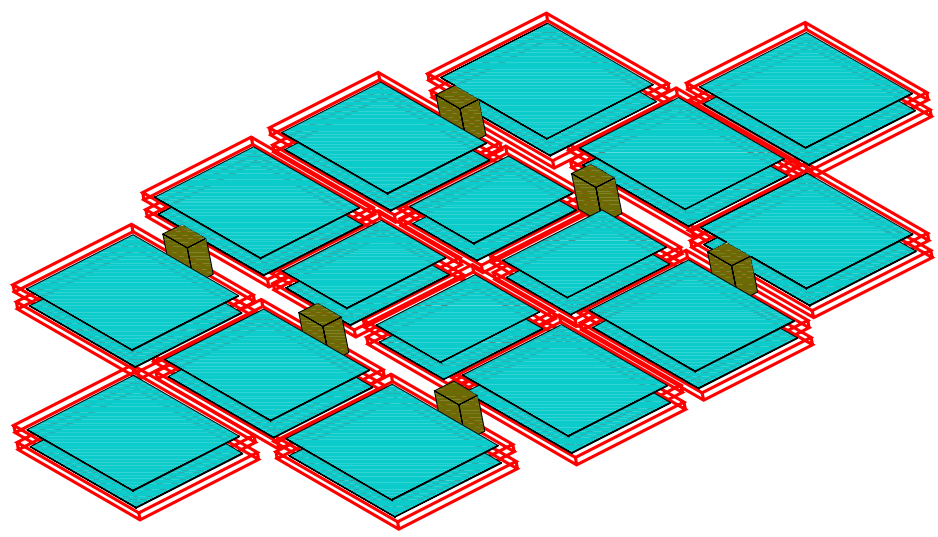

sensitive area: $2 \times 122 \mathrm{~m}^{2}$ area of basement: $20 \times 16 \mathrm{~m}^{2}$

Figure 2: Two MWPC layers installed in the basement of the central detector. 


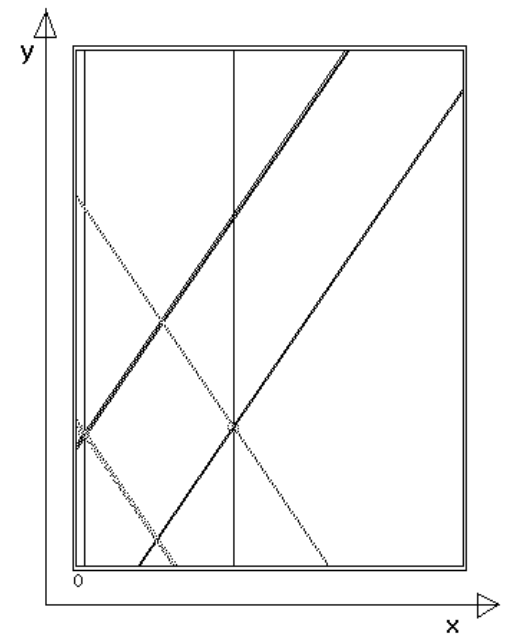

Figure 3: Anode wires and diagonal cathode strips are used to determine the particle position.

point of hit particles [10]. The rectangular MWPC have three different sizes with sensitive areas of ca. $5 \mathrm{~m}^{2}, 8.2 \mathrm{~m}^{2}$, and $8.8 \mathrm{~m}^{2}$, respectively (Fig.2). Tracking routines reconstruct position and inclination of penetrating muons with an accuracy of about $1^{\circ}$.

One active layer of the calorimeter setup in the third gap from the top (shielded by ca. 30 r.l.) is a layer of 228 plastic scintillator modules, building a fast trigger for the central detector devices and a timing facility enabling the measurement of arrival time distributions [11] of particles penetrating through the absorber above. Each module contains two scintillation detectors with an area of $0.9 \mathrm{~m}^{2}$ and is read out by a wavelengthshifter and a single photomultiplier. The sensitive coverage of this trigger layer system is about $64 \%$ of the total central detector area.

The top cluster (Fig.1) is an array built up of 25 detector boxes, similar to those of the trigger layer. It is operated to close the gap of the field array for measurements of the density and the arrival times of the charged-particles component.

For detailed studies of the detector efficiencies and responses first a set of EAS, simulated for the KASCADE observation level (110 m a.s.l.), has been prepared using the Monte Carlo air shower simulation program CORSIKA [12] (Vers.5.2), which includes GHEISHA [13] and different packages of high-energy interaction models like VENUS [14] and QGSJET [15]. The electron-photon component is also simulated by the Monte Carlo procedure of EGS4 [16]. The influence of the Earths magnetic field is taken into account.

The simulation calculations cover the energy range of $10^{14} \mathrm{eV}-10^{16} \mathrm{eV}$ for five different mass groups of the primaries with isotropic incidence: $\mathrm{H}=$ proton, $\mathrm{He}=$ alpha particles, $\mathrm{O}=\mathrm{CNO}$ group, $\mathrm{Si}=$ heavy group, $\mathrm{Fe}=$ very heavy group.

The response of all KASCADE detector systems to the EAS components has been determined by simulations using the GEANT code [17], a well established tool in high-energy physics for the simulation of particle detector properties. The particles 
of the generated EAS are tracked with varying core distances from the central detector (up to $20 \mathrm{~m}$ ). All relevant physical interactions of particles with matter are taken into account, and each produced secondary particle is included in the further tracking procedures. The output of the simulations is stored in the same way as measured data, therefore measured and simulated data can be reconstructed with the same procedures, thus minimizing systematic errors in comparisons.

\section{Measurements and Reconstructed Parameters}

The present data set stems from an acquisition period of more than 12 months, comprising more than 20 millions of shower events with all KASCADE detector systems simultaneously triggered by the charged-particle component, observed by the field array and subsequently readout and stored. For the analysis, presented in this work the relevant parameters reconstructed for each registered shower from the data of the field array are: the core location, the arrival direction of the shower, the shower size $N_{e}$ (total number of electrons), the total number of muons $N_{\mu}$, and the so-called truncated muon number $N_{\mu}^{t r}$. The latter is the content of muons with $E_{\mu}>300 \mathrm{MeV}$ in the limited range of the lateral distribution in the distance of $40 \mathrm{~m}-200 \mathrm{~m}$ from the shower center. EAS simulations have revealed that $N_{\mu}^{t r}$ is a nearly mass independent identifier for the primary energy $E_{\text {prim }}$ of the shower for the KASCADE observation level. The reconstruction accuracy of high-energy central showers for these observables are in the range of $10-20 \%$ for the estimated particle numbers, ca. $2 \mathrm{~m}$ for the core location, and ca. $0.5^{\circ}$ for the arrival direction.

From the data measured by the hadron calorimeter reconstructed parameters are the number of hadrons above $20 \mathrm{GeV}$ in an area of $16 \times 16 \mathrm{~m}^{2}$, their total energy and the energy of the hadron with maximum energy. Up to now these "hadronic" shower parameters have not been introduced in our analysis but will be used for comparisons.

The reconstructed parameters describing the trigger layer information are the sum of the deposited energy, measured with the scintillation detectors, and the number of hitted detectors.

The reconstruction of the data from the MWPC system provides the tracks of muons above a threshold of $2 \mathrm{GeV}$ and a hit pattern: number and spatial distribution of muons and of produced secondaries. In particular, showers of high energy with the core located in the central detector exhibit a large number of secondaries generated by muonic and hadronic interactions in the absorber above. Thus the observation with the MWPC includes also some spectroscopic information.

On the basis of these shower parameters the data sample has been enriched with data from the central region of high-energy showers by cuts of $N_{\mu}^{t r}$ and of the estimated core location. Additionally a selection along the deposited energy in the trigger plane is used to improve the rejection of low energy showers. The effects of the cuts have been studied and controlled by shower simulations for each mass group, and the efficiencies of the selections are determined separately for the different primary masses of different energies $E_{\text {prim }}$, with data registered in various radial bins of the distance $R_{\text {core }}$ from the center of the central detector. Figure 4 displays the selection efficiencies for the case of iron and proton induced showers. Due to the 

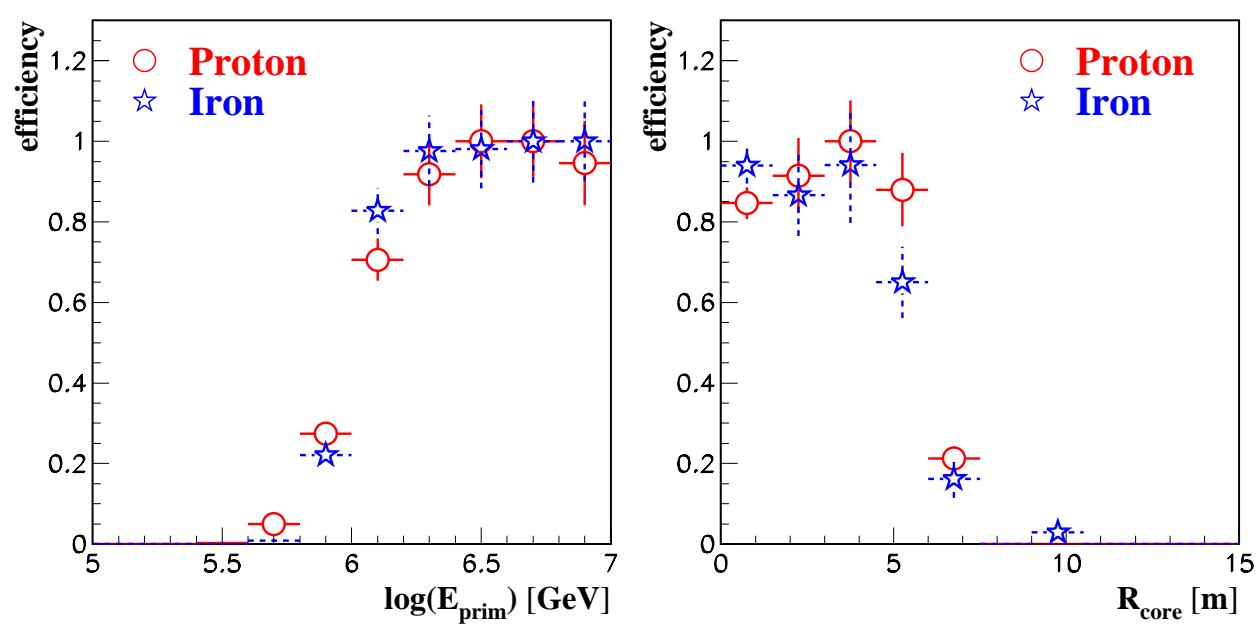

Figure 4: Selection efficiencies for proton and iron induced showers in different bins of primary energy and core distance.

larger fluctuations in the shower development of proton induced showers the selection efficiencies for energies smaller than $10^{15} \mathrm{eV}$ are slightly increased for proton induced showers as compared to those of iron primaries.

After the selection procedure the data sample shrinks to 1411 real measured showers with a distance $R_{\text {core }}<5 \mathrm{~m}$ and with energies larger than ca. $10^{15} \mathrm{eV}$ at disposal for the further analysis.

\section{Multifractal Moments Analysis of the Structure of the Shower Core}

Figure 5 shows lateral distributions of the muon and hadron components of highenergy showers from Monte Carlo simulations, and it displays the differences in the central part observable in first $8 \mathrm{~m}$ from the shower center. Showers induced by light primaries exhibit a steeper lateral distribution of both particle components than showers of heavy primaries, which contain less particles and with comparatively weaker energy spectra. These feature can be measured with the MWPC systems registrating muons of energies above $2 \mathrm{GeV}$ and high-energy hadrons indicated by the secondaries produced in the absorber. Hit density distributions of two typical measured shower cores are shown in Figure 6. As simulation demonstrate the hits are not only due to traversing muons, they arise also from $\delta$-electrons and small electromagnetic showers induced by muon interactions and cascading electrons. Large hit clusters in the MWPC displays originate from tails of hadronic cascades of hadrons of energies $>10 \mathrm{TeV}$, from hadronic secondaries like neutrons and nuclear interactions of muons. All these effects are mapping the differences in energy and lateral distributions for different primaries. The correlation of the energy and the distance from the shower center (radius R) of high-energy hadrons from 200 simulated proton and iron induced showers (with the energy $E_{\text {prim }}>10^{15} \mathrm{eV}$, and the spectral distribution $\sim E^{-3.0}$ ) is shown in Figure 7. Obviously the proton 

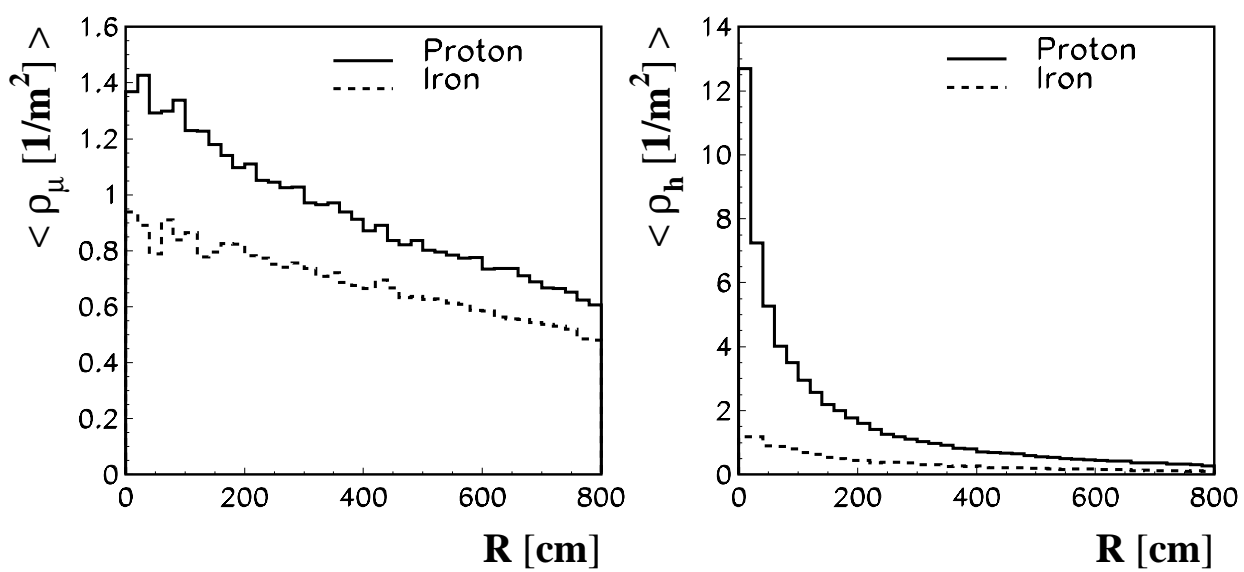

Figure 5: Average muon (left) and hadron (right) lateral distributions $\left(E_{\mu, h}>\right.$ $2 \mathrm{GeV}$ ) of proton and iron induced showers in the region of $8 \mathrm{~m}$ from the shower center (CORSIKA simulations taking into account the energy spectrum above 1 PeV with an isotropic shower incidence).

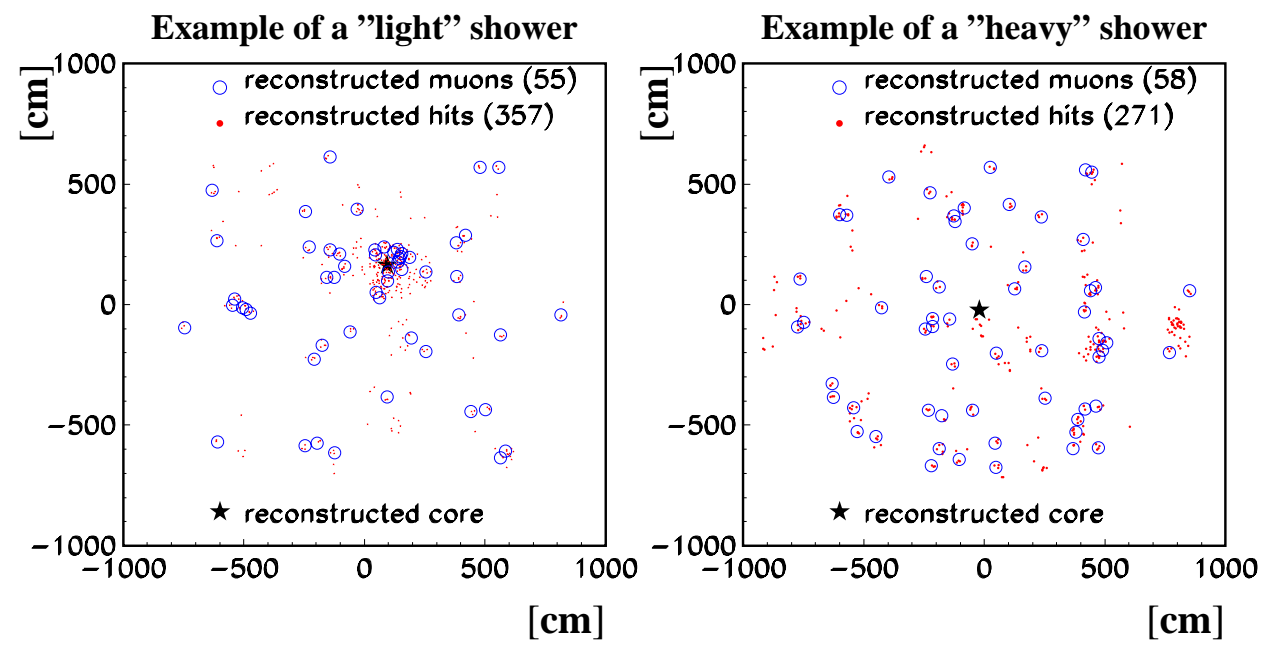

Figure 6: Examples of typical hit density distributions measured by the MWPC system. The stars mark the core of the showers estimated by the KASCADE field array.

induced showers are more concentrated in the innermost part of the core, with large clusters reflecting the steeper distribution with more energetic particles. Considering single showers, however, the fluctuations of the lateral density distributions are enormous and prevent a sufficiently accurate determination of the core by adjusting a prechosen lateral distribution function to the observed hits. However, there are well-established mathematical methods of analysis to reveal from seemingly irregular (and noisy) distributions, which are not completely due to statistics, specific features which are characteristic for the origin of the observed patterns. Under certain conditions a parametrisation in terms of multifractal moments [18] has a reasonable basis. Studying patterns of simulated EAS it has been demonstrated, just in an empirical way of a successful application for re-identification of the studied EAS, 


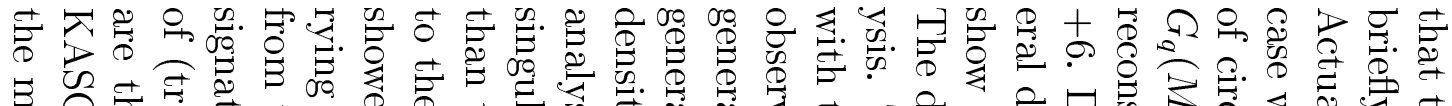

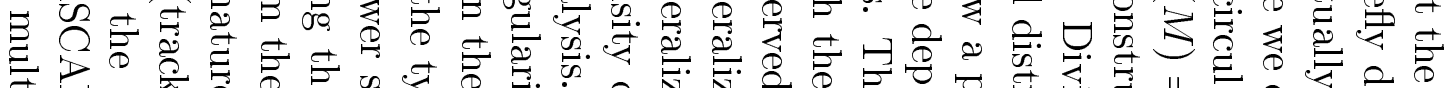
寻 $\begin{aligned} & 0 \\ & 0\end{aligned}$

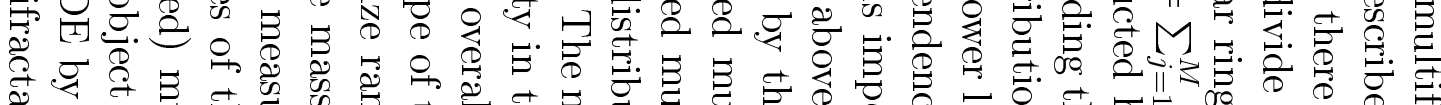

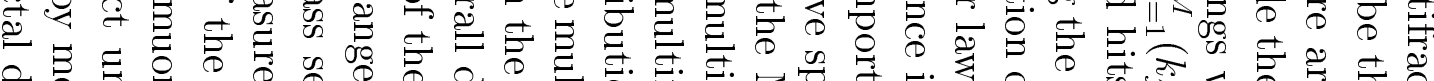
. จ

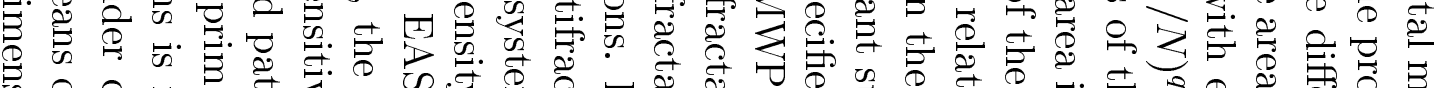

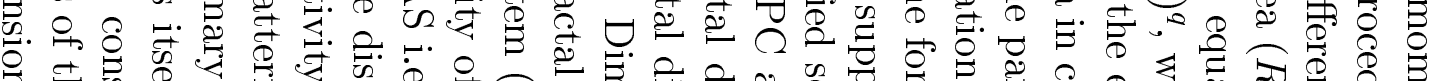

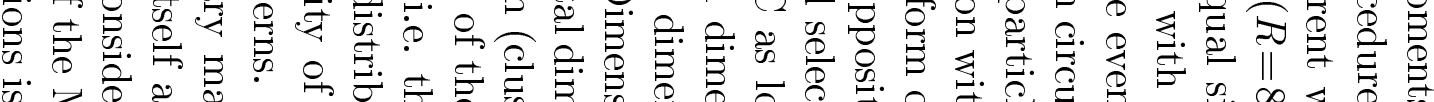
乡.

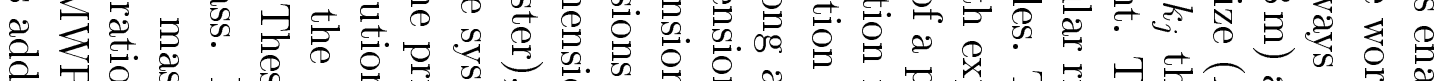

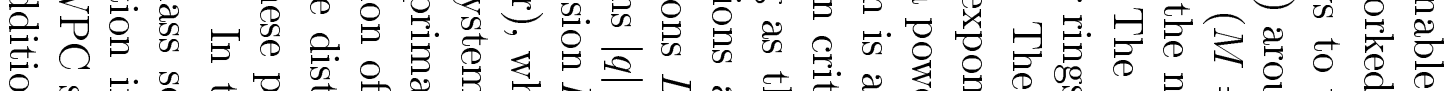

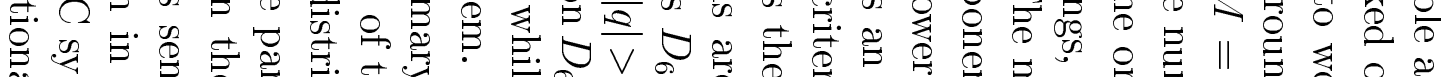

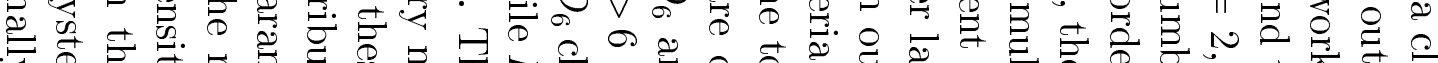

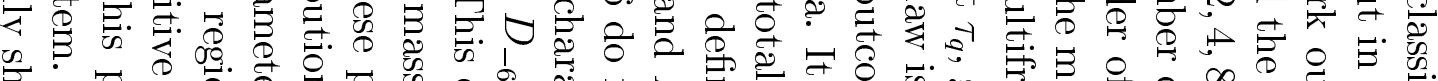

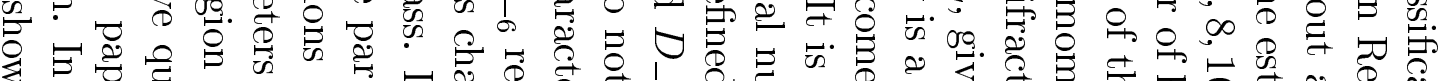

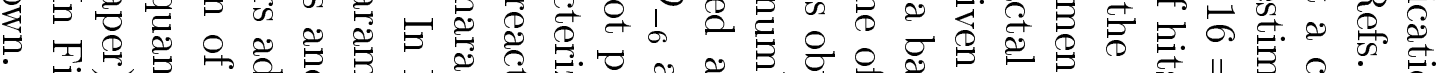

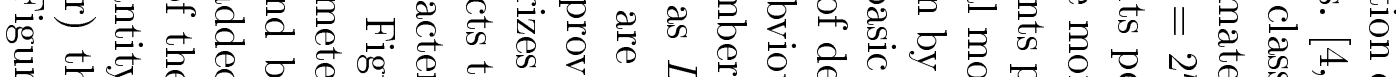

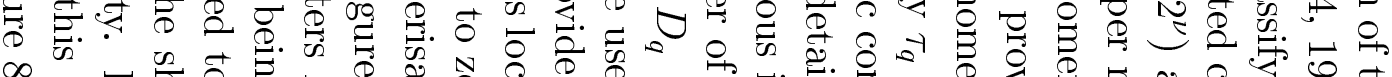

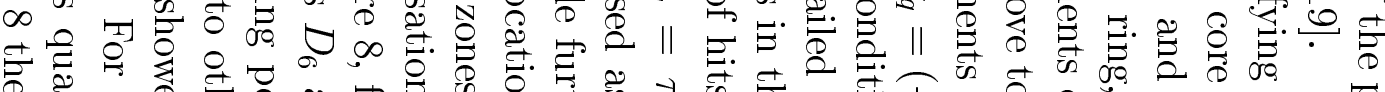

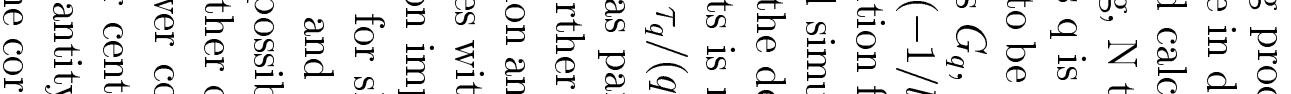
ब.

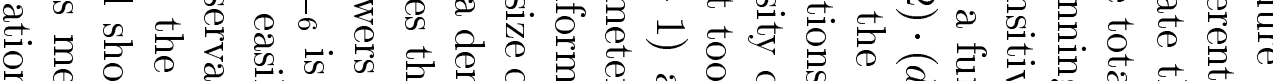

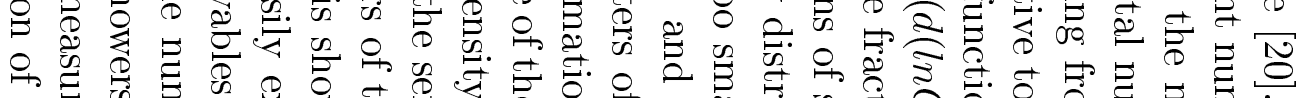

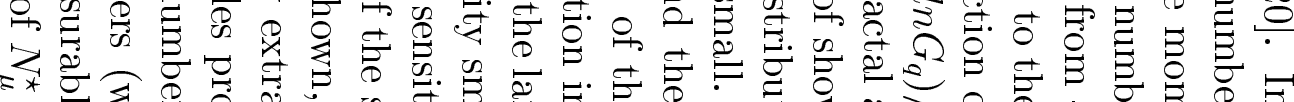

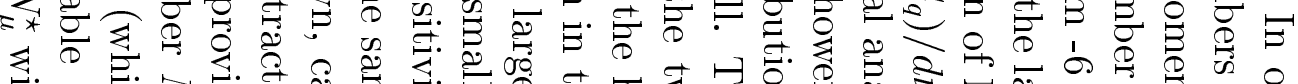

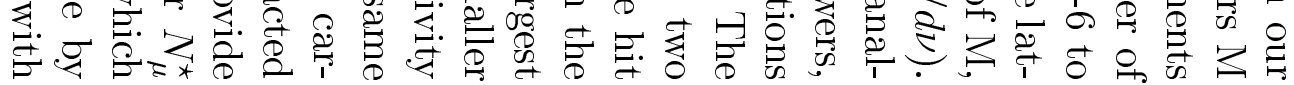

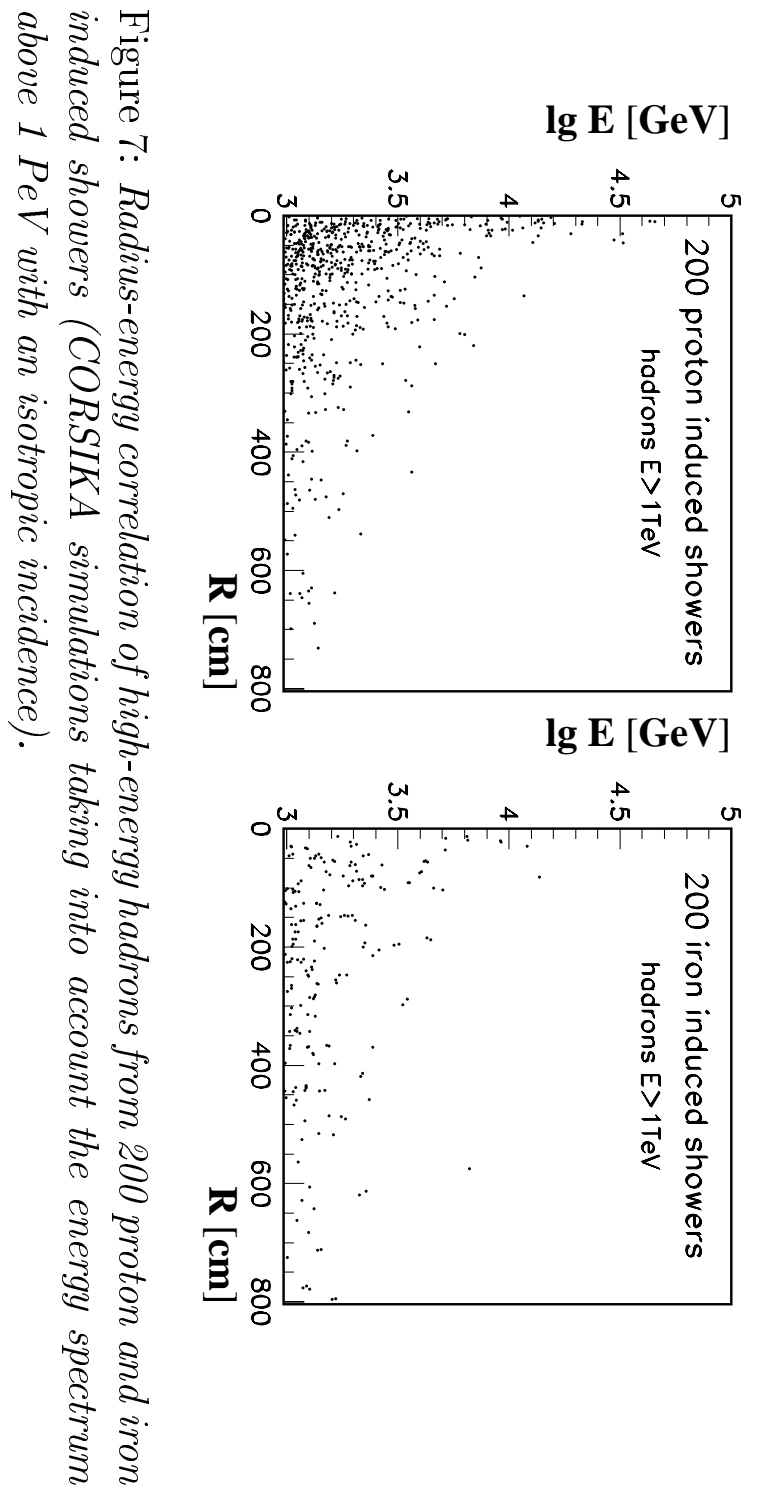



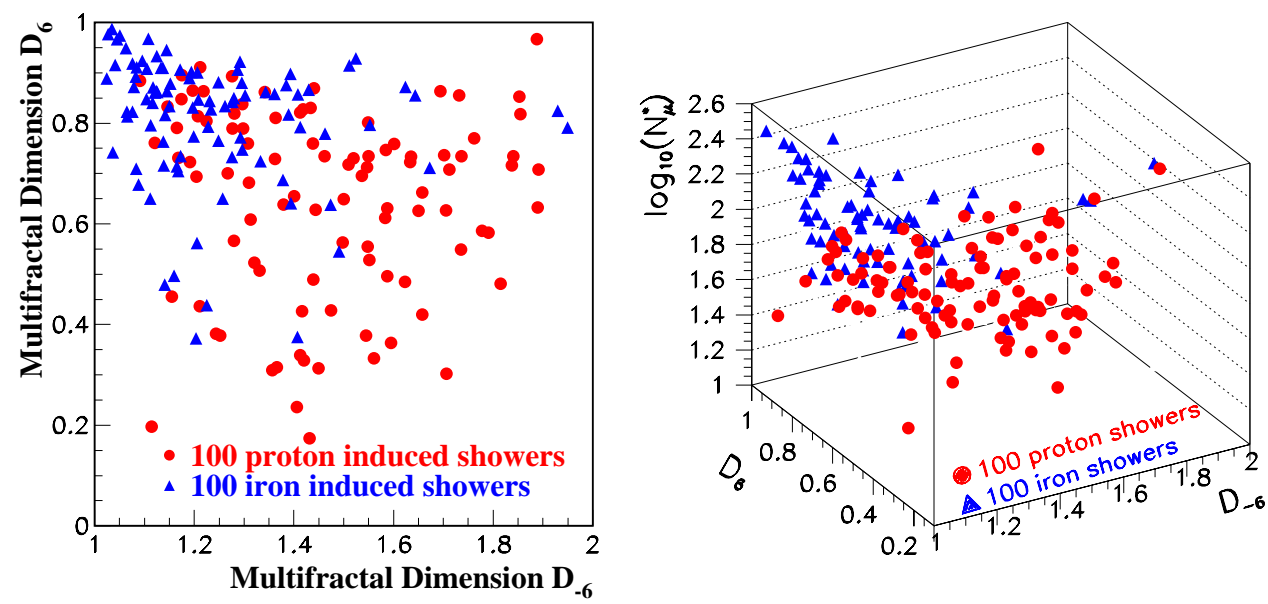

Figure 8: Sensitivity of the two multifractal dimensions $D_{-6}$ and $D_{6}$ and number of tracked muons in the core $\left(N_{\mu}^{\star}\right)$ for mass separation for showers of ca. identical shower sizes $\left(4.7>\log N_{e}<5.5\right)$. Each point or triangle represents a single shower in the parameter space.

\section{Analysis of the Measured Data}

Using five experimentally determined mass sensitive observables for each shower (shower size $N_{e}$, number of tracked muons in the MWPC system $N_{\mu}^{\star}$, generalized multifractal dimensions $D_{6}$ and $D_{-6}$, and the zenith angle of the shower direction $\Theta)$ as input parameters, a simple artificial neural network is constructed [21]. The net reduces the five parameters to one parameter $\mathrm{y}_{\mathrm{NN}}$, representing the mass discrimination, with output values between zero and one. This net (configured as a feed-forward net with back propagation) is trained by simulated proton and iron showers, which are generated on the basis of the VENUS model, fulfilling the above explained selection cuts and following a power law spectrum $\sim E^{-3.0}$ and an isotropical distribution of the arrival directions up to $40^{\circ}$. For proton induced showers a "true value" zero is assumed, for iron induced showers this start value is one. After the training all thresholds and weights of the net are fixed, and independent samples of simulated and the measured showers are classified according to the "mass parameter" $\mathrm{y}_{\mathrm{NN}}$. Figure 9 compares the $\mathrm{y}_{\mathrm{NN}}$ - distributions of the measured data with that of simulated showers of different primaries and two different interaction models. Under the assumption of mass independent full efficiency and energy independence of the distribution (which is both fulfilled after applying the selection criteria) the simulated $\mathrm{y}_{\mathrm{NN}}$ distributions for each particular one-component composition are compared with the data.

For exploring the information about the mass composition, in a first simplified approach the $\mathrm{y}_{\mathrm{NN}}$ distributions are further specified by different ranges of the truncated muon number ( $\propto$ primary energy) and compared to the variation of the average of the $\mathrm{y}_{\mathrm{NN}}$ distributions. Figures 10 and 11 show the variation with the truncated muon number and with the shower size, respectively, for two different simulated pri- 


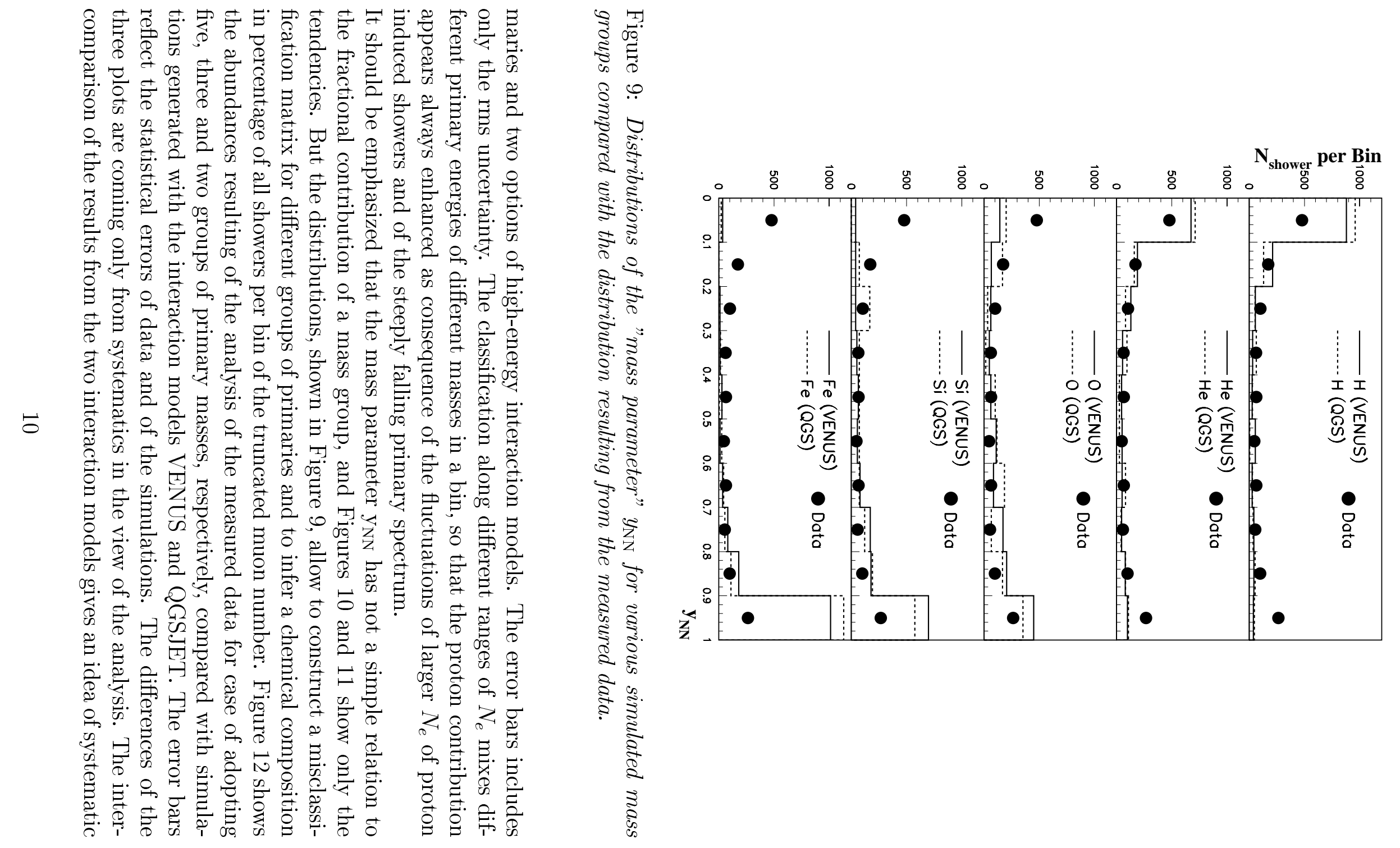




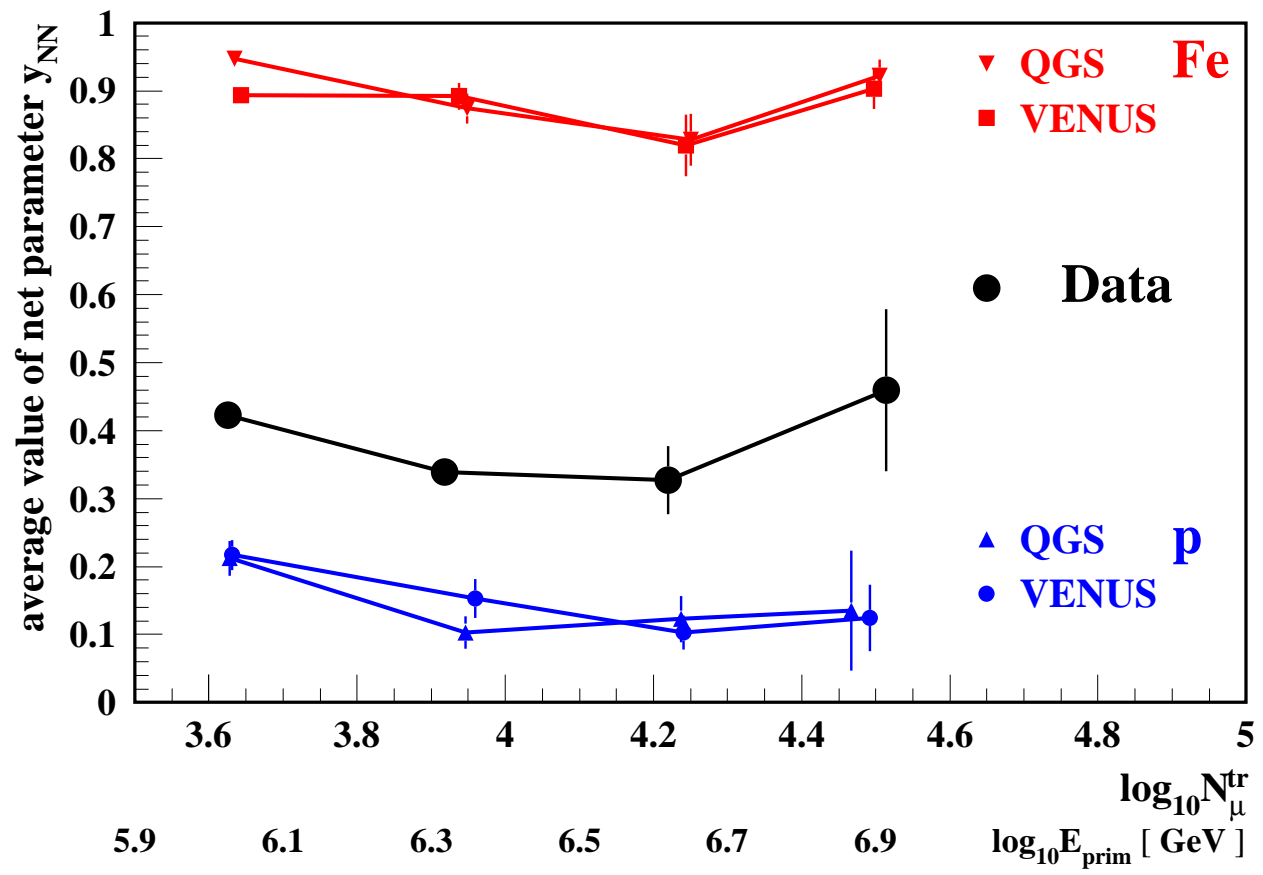

Figure 10: Variation of the average value of the net parameter $y_{\mathrm{NN}}$ with the truncated muon number of proton and iron induced simulated EAS compared with $y_{\mathrm{NN}}$ from data. In the lower abscissa the truncated muon number is related to the primary energy (of showers with zenith angles of $20^{\circ}$ ). The knee is assigned to $\log _{10} E_{\text {prim }}[G e V] \approx 6.5$.

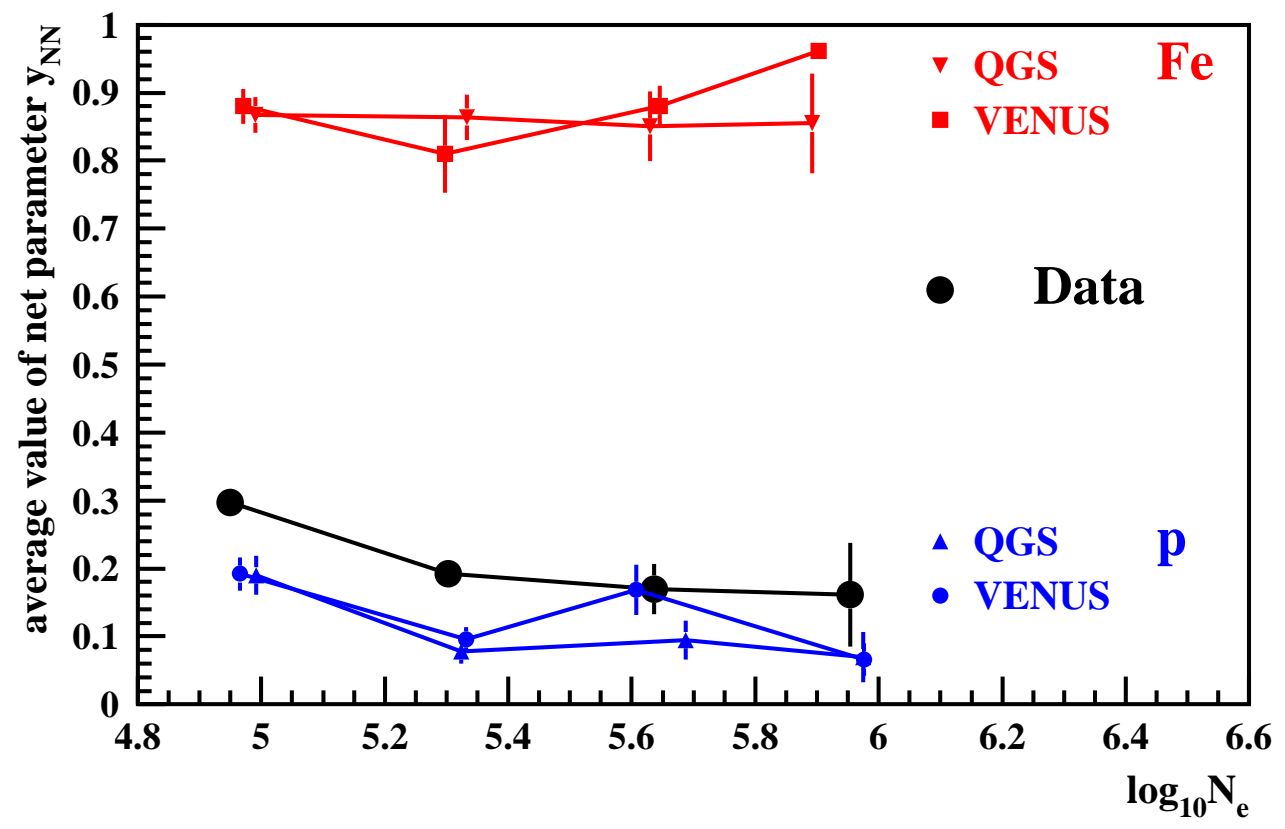

Figure 11: Variation of the average value of the net parameter $y_{\mathrm{NN}}$ with the shower size of proton and iron induced simulated EAS compared with measured data. 
VENUS
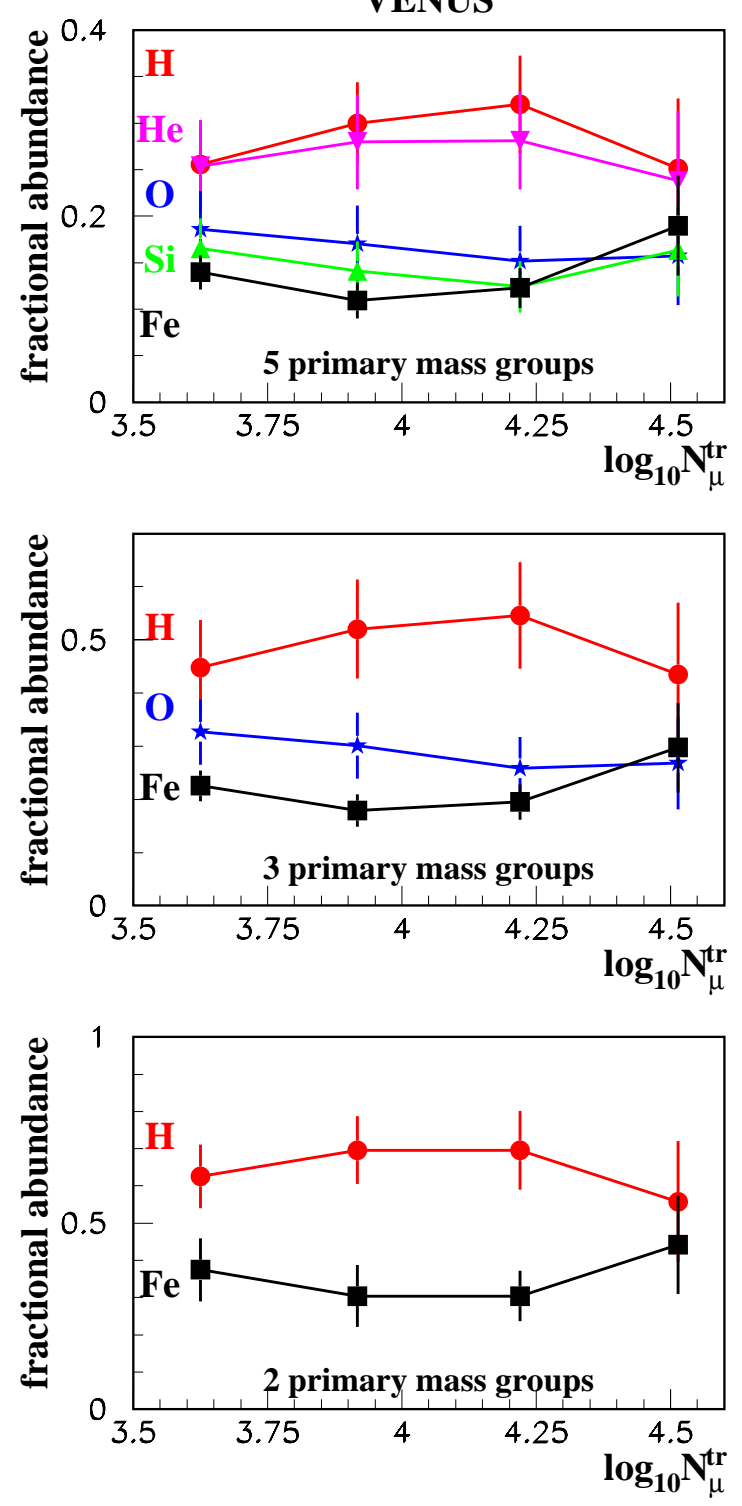

QGSJET
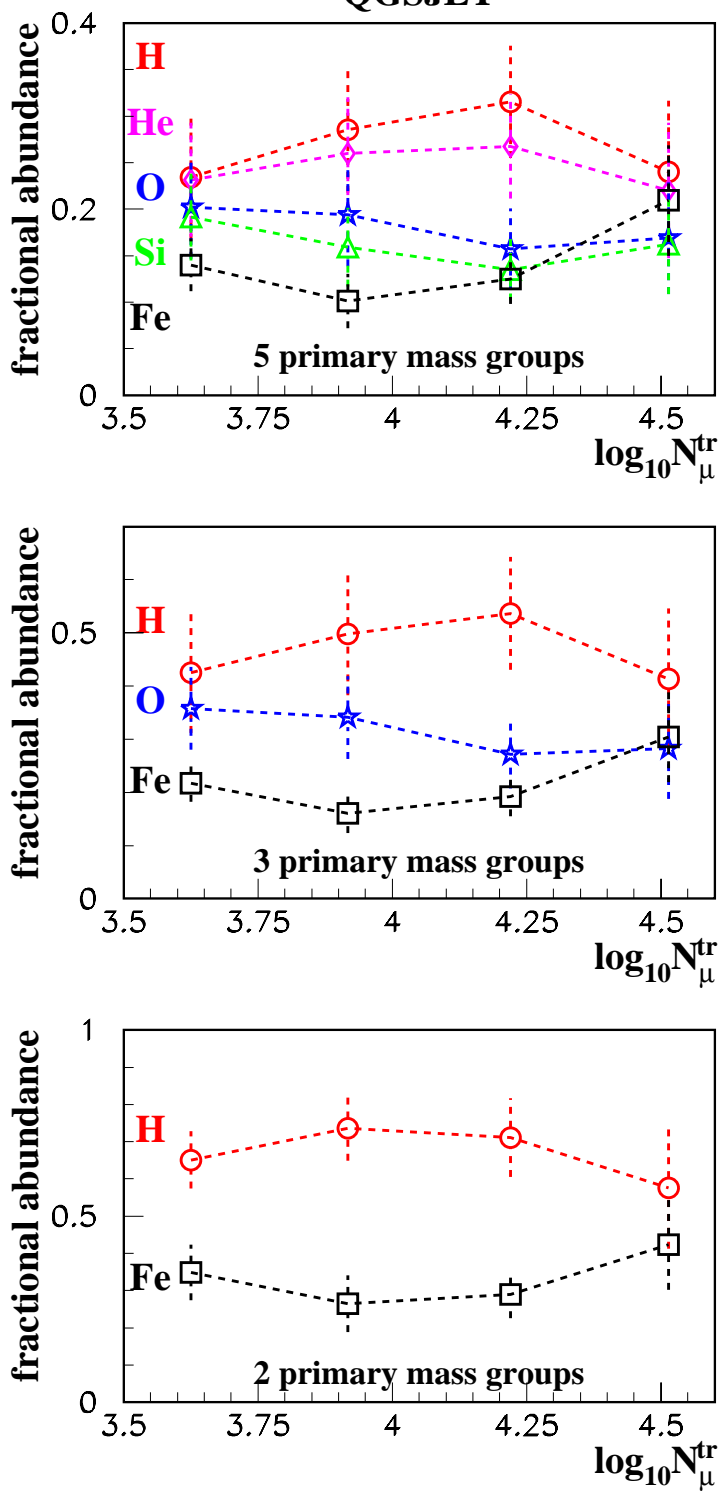

Figure 12: Relative abundances of different mass groups of primary cosmic rays in the energy region around the knee $\left(\log _{10} N_{\mu}^{t r} \approx 4.1\right)$, analysed on the basis of the interaction models VENUS and QGSJET.

errors due to model dependence. Uncertainties depending from the chosen number of primary groups are of higher order and may indicate the mass resolution. For this analysis the net is trained only with two groups which implies the tendency of the net to classify each shower either to proton or to iron induced. So the three medium groups differ only in the average value of the net output parameter from proton and iron, respectively, and do not peak inside on a specific value of $\mathrm{y}_{\mathrm{NN}}$. Promising is the fact, that in all three cases of Figure 12 the tendency of the mass variation around the knee region remains stable. 


\section{Spectral Variations of Shower Samples of Dif- ferent Mass Classifications}

The method of the multifractal moments analysis of the hit patterns of shower cores measured with the MWPC system of the KASCADE central detector allows to classify the measured EAS in different subgroups, e.g in a p-like (with $\mathrm{y}_{\mathrm{NN}}<0.2$ )

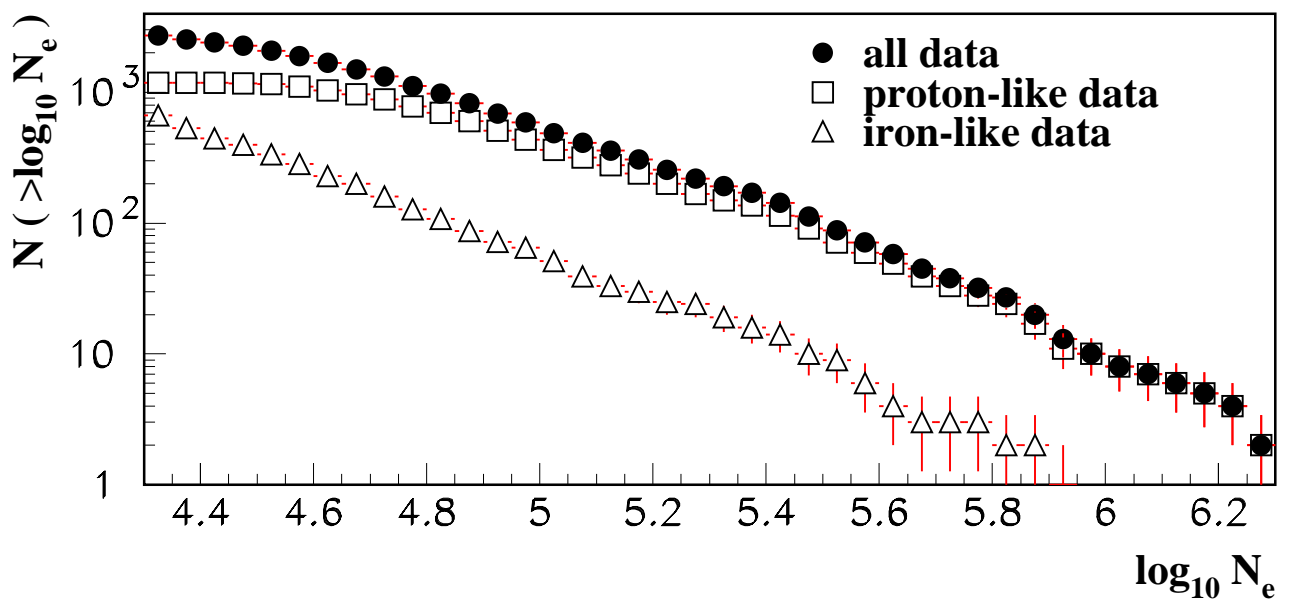

Figure 13: Integral spectra of the shower size $N_{e}$ for all, for proton-like and for iron-like measured showers.

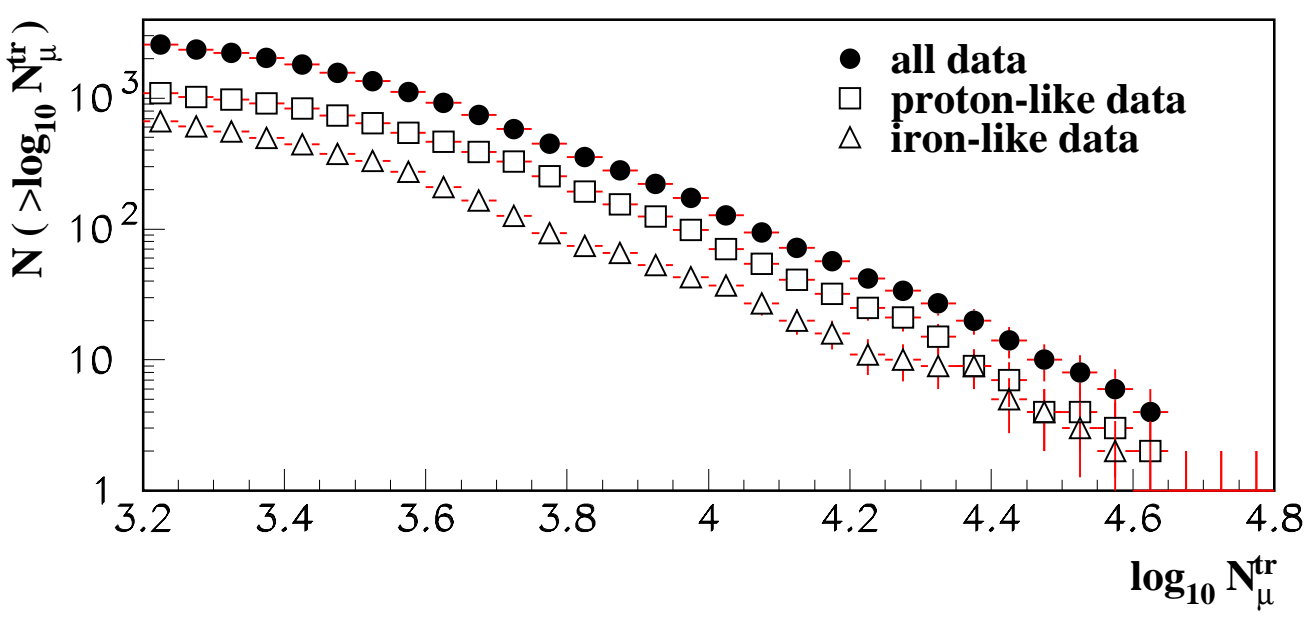

Figure 14: Integral spectra of the truncated number of muons $N_{\mu}^{t r}$ for all, for protonlike and for iron-like measured showers.

and a Fe-like $\left(\mathrm{y}_{\mathrm{NN}}>0.8\right)$ group. With such samples integral spectra along different shower parameters can be studied for light- and heavy-primary induced showers. Such integral spectra resulting from a reconstruction of the shower parameters of each individual shower are shown in Figures 13-16: 


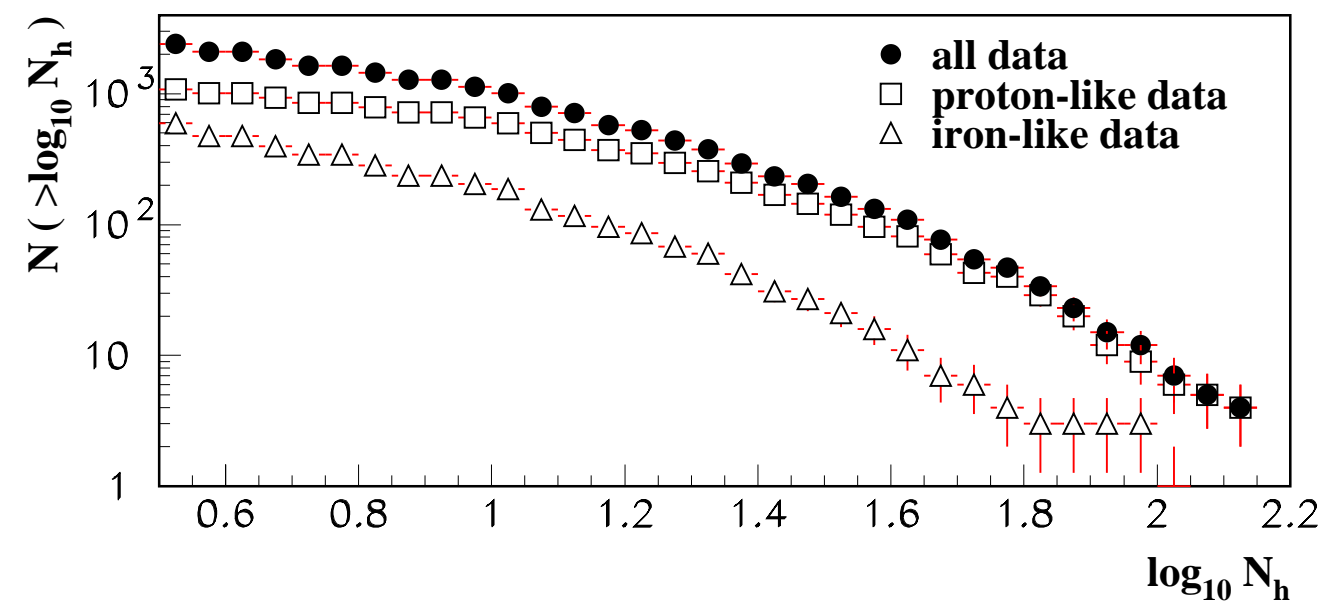

Figure 15: Integral spectra of the total number of hadrons $N_{h}\left(E_{h}>20 \mathrm{GeV}\right)$ registered in the calorimeter for all, for proton-like and for iron-like measured showers.

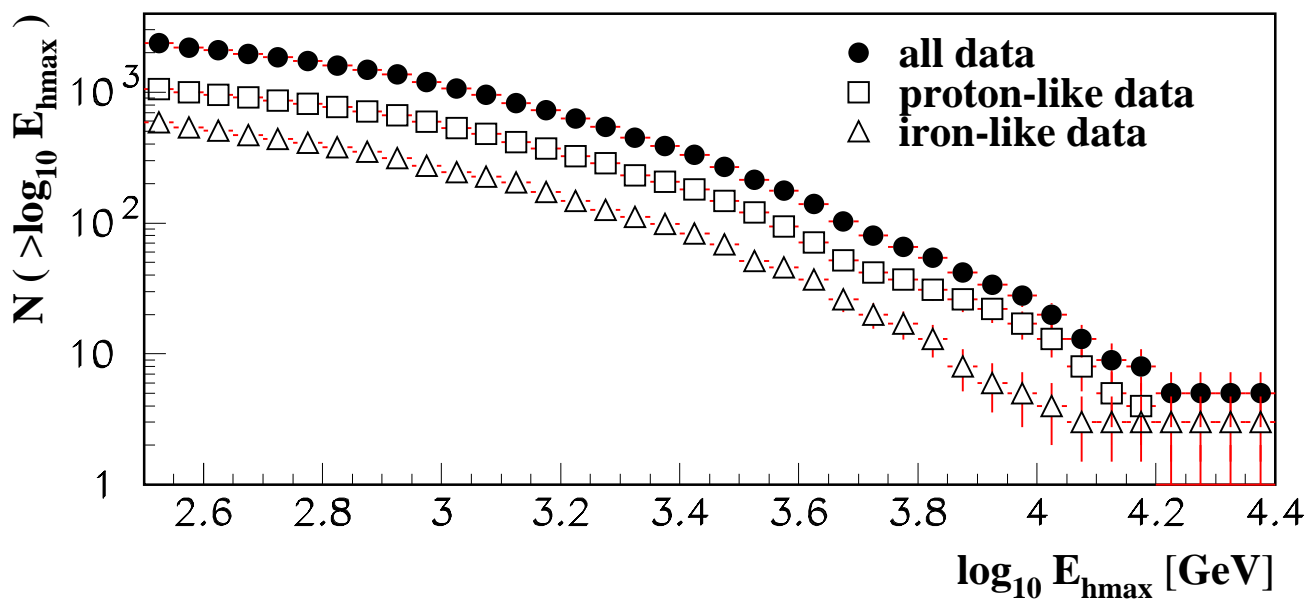

Figure 16: Integral spectra of the energy $E_{\text {hmax }}$ of the leading hadron in the shower for all, for proton-like and for iron-like measured showers.

- shower size spectrum $N_{e}$ (Fig.13)

- spectrum of the truncated muon number (40-200 m) $N_{\mu}^{t r}$ (Fig.14)

- distribution of the number of hadrons $\left(E_{h}>20 \mathrm{GeV}\right)$ reconstructed in the calorimeter $N_{h}$ (Fig.15)

- distribution of the hadrons with the highest energy $E_{h \max }$ in the showers (Fig.16) 
The slopes of the "all data" distributions (spectral indices) are in good agreement with results of all KASCADE showers extracted with much higher statistical accuracy [22].

With the above remark to Figure 11 about the influence of the $N_{e}$ fluctuations of proton induced showers, it is less surprising that the $N_{e}$ spectra are dominated by the proton-like showers, increasingly with increasing $N_{e}$ (Fig.13). A similar argument holds for the $N_{h}$ spectra (Fig15). The distributions along $N_{\mu}^{t r}$ and $E_{h \max }$, which are less affected by the energy mixing, tentatively show an approximately constant ratio of the proton-like to the iron-like contributions, and though with large statis-

\begin{tabular}{|c|c|c|c|c|}
\hline & \multicolumn{2}{|c|}{$"$ p-like" $\left(\mathrm{y}_{\mathrm{NN}}<0.2\right)$} & \multicolumn{2}{|c|}{ "Fe-like" $\left(\mathrm{y}_{\mathrm{NN}}>0.8\right)$} \\
\hline & VENUS & QGSJET & VENUS & QGSJET \\
\hline Protons & $77 \%$ & $77 \%$ & $6 \%$ & $7 \%$ \\
\hline Alpha & $61 \%$ & $62 \%$ & $13 \%$ & $13 \%$ \\
\hline Medium & $15 \%$ & $26 \%$ & $47 \%$ & $37 \%$ \\
\hline Heavy & $6 \%$ & $5 \%$ & $62 \%$ & $54 \%$ \\
\hline Iron & $2 \%$ & $2 \%$ & $85 \%$ & $88 \%$ \\
\hline & \multicolumn{2}{|c|}{ "light" $\left(\mathrm{y}_{\mathrm{NN}}<0.5\right)$} & \multicolumn{2}{|c|}{ "heavy" $\left(\mathrm{y}_{\mathrm{NN}}>0.5\right)$} \\
\hline & VENUS & QGSJET & VENUS & QGSJET \\
\hline Protons & $87 \%$ & $88 \%$ & $13 \%$ & $12 \%$ \\
\hline Alpha & $77 \%$ & $76 \%$ & $23 \%$ & $24 \%$ \\
\hline Medium & $27 \%$ & $37 \%$ & $73 \%$ & $63 \%$ \\
\hline Heavy & $16 \%$ & $27 \%$ & $84 \%$ & $73 \%$ \\
\hline Iron & $6 \%$ & $3 \%$ & $94 \%$ & $97 \%$ \\
\hline
\end{tabular}

Table 1: Classification rate of simulated events of the fractal moments classification.

tical uncertainty, even a slight increase of the heavy fraction at the highest energies (Figs.14, 16) may be indicated.

In order to estimate the misclassification probability of light and heavy showers by classifying according to $\mathrm{y}_{\mathrm{NN}}<0.2$ and $\mathrm{y}_{\mathrm{NN}}>0.8$, respectively, the simulated EAS (used in Figure 9 from two different generators) have been re-identified by the classification procedure. Table 1 gives the composition of the two samples by the percentage of true- and re-identified showers. These numbers show that the methods of fractal moments allows to select from the observed EAS reliable samples of dominantly light and heavy composition, respectively, with uncertainties in the range of the statistical error (except at the highest energies). So we may have good confidence in the tendencies revealed with the spectra.

The indices of the spectra does not alter significally, if the samples of the showers are defined by another choice of $\mathrm{y}_{\mathrm{NN}}$. In Figure 17 the integral spectra of truncated muon number is shown for all and for heavy and light induced showers with the condition of $\mathrm{y}_{\mathrm{NN}}>0.5$ and $\mathrm{y}_{\mathrm{NN}}<0.5$, respectively. Corresponding re-identification is shown in the lower part of Table 1.

In the analysis of the $N_{e}-N_{\mu}$ correlation of data taken with the KASCADE field 


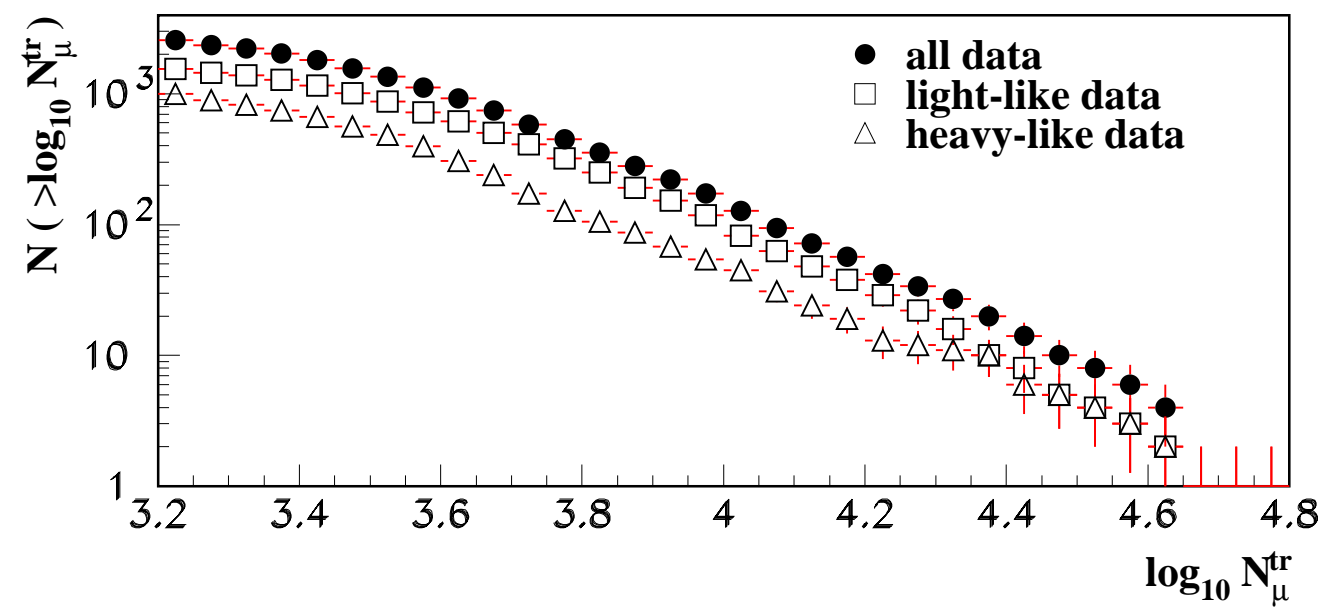

Figure 17: Integral spectra of the truncated number of muons $N_{\mu}^{t r}$ for all, for light-like $\left(y_{\mathrm{NN}} \leq 0.5\right)$ and for heavy-like $\left(y_{\mathrm{NN}}>0.5\right)$ measured showers.

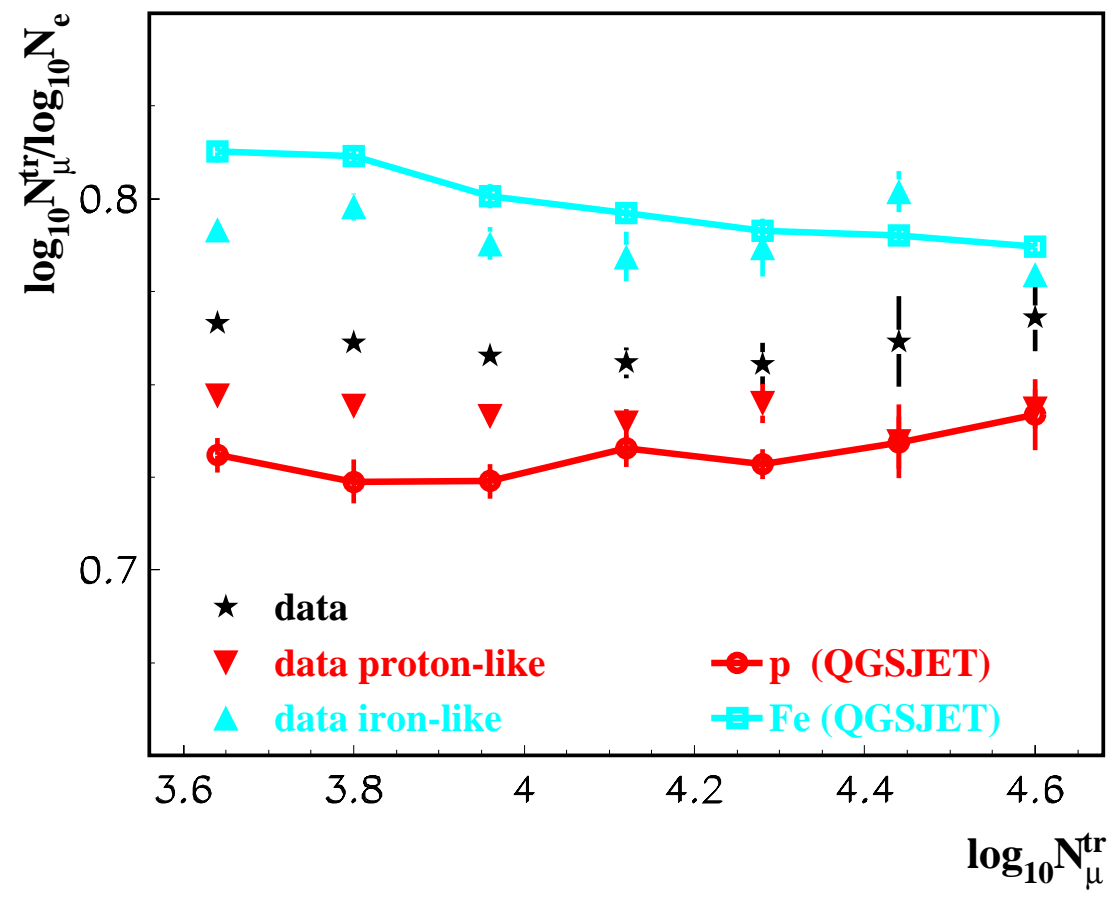

Figure 18: The $N_{\mu}^{t r} / N_{e}$ ratio variation for all data, for proton-like and for iron-like measured data in comparison with simulated proton and iron induced showers.

array, the ratio $\log _{10} N_{\mu}^{t r} / \log _{10} N_{e}$ vs. $\log _{10} N_{\mu}^{t r}$ has been assumed as an indication of the energy-dependent variation of the composition. We use this presentation to show this variation within the samples of showers observed with the central detector, and classified along the appearance of the shower core. The result does in fact confirm the classification procedure (Fig.18). 


\section{Conclusions}

The present investigation uses the method and procedures, suggested and worked out in [4] for the exploration of the appearance of the EAS core in view of information on the mass of the primary. They were applied to a sample of experimental data, measured with the KASCADE central detector and carrying information about the energy and lateral distributions of the muonic and hadronic EAS components in the shower center. The method is based on the fact that the seemingly irregular patterns of hits, observed with the position-sensitive MPWC installed under the iron calorimeter, contain mass discriminating signatures. They can be characterised in terms of multifractal moments of the distributions. The multifractal analysis is able to reveal the systematic physical fluctuations, which are superimposed to the statistical fluctuations in the density distributions. It quantifies the information by two parameters, which can be added to other relevant shower parameters and condensed in one single mass-sensitive output parameter $\mathrm{y}_{\mathrm{NN}}$ of a neural network for a multidimensional analysis. It should be remarked that the number of muons $N_{\mu}^{\star}$ registered in the shower center, is additionally indicative for the primary mass, in contrast to the truncated muon number $N_{\mu}^{t r}$, used as measurable parameter of the primary energy at the KASCADE measurements. The procedures follow the general scheme to compare "theoretical" data, constructed from Monte Carlo simulations of the EAS development and of the detector response with real data by identical reconstruction procedures of shower variables, in an event-by-event analysis.

In the actual status of data collection of KASCADE the sample of showers whose cores are identified in the central detector is still rather small, so that the resulting statistical accuracy remains limited, in particular for high-energy EAS. This is in addition to the limitations due to a restricted number of simulations. Thus the present procedures use some simplifications, e.g. the training of the net with only two mass groups, affecting the mass resolution. However, the way to a more detailed analysis of a larger data sample is traced.

The results, first by classifying the individual showers and dividing in various mass groups, then calculating the relative mass fractions, reveal the tendency of the variation of the mass composition around the knee region. It is in agreement with the tentative results from the $N_{e^{-}} N_{\mu}$ correlation of KASCADE and other data [22, 23], suggesting a lighter composition in a narrow band near the knee with an increase of the heavy component just beyond. Of course, due to still unexplored systematic errors and lack of statistical accuracy a discussion of the results in context of models of the origin of the knee $[24,25,26]$ is premature.

The procedure classifying the measured EAS by a mass sensitive parameter (the output of a neural net trained with an adequate sample of simulated showers) provides the possibility to create experimental shower samples of predominantly heavy and predominantly light primaries, respectively, to be used for further empirical studies of the distributions of various shower variables. Such studies may reveal characteristic features and differences of the EAS parameters of different mass primaries and provide further tests of Monte Carlo simulations, independent from the question of cosmic ray mass composition. 


\section{Acknowledgments}

We would like to thank M. Elzer, H. Leibrock, and K. Köhler for their help in operating the proportional chambers during the data acquisition.

The collaborating group from the Cosmic Ray Division of the Soltan Institute of Nuclear Studies in Lodz and from the University of Lodz is supported by the Polish State Committee for Scientific Research (grant No. 2P03B 160 12). The work is partly supported by the research grant 94964 of Armenian Government and by grant A116ISTC as well as by a grant of the Romanian Ministry of Research and Technology. The KASCADE collaboration work is embedded in the frame of scientifictechnical cooperation (WTZ) projects between Germany and Poland (Nr.92-94), Armenia (X131.2), and Romania (Rum-002-94).

\section{References}

[1] N. N. Kalmykov, G. B. Khristiansen, Journal Phys. G 21 (1995) 1279

[2] B. Wiebel-Sooth, P. L. Biermann, H. Meyer, Astron. Astrophys. 330 (1998) 389

[3] H. O. Klages et al. - KASCADE Collaboration, Nucl.Phys. (Proc.Suppl.) 52B (1997) 92

G. Schatz et al. - KASCADE Collaboration, Nucl.Phys. (Proc.Suppl.) 60B (1998) 151

[4] A. Haungs, J. Kempa, H. Rebel, H. J. Mathes, J. Wentz, Nucl. Instrum. and Meth. A 372 (1996) 515

[5] B. B. Mandelbrot, Journ. Fluid Mech. 62 (1974) 331

[6] R. C. Hwa, Phys. Rev. D41 (1990) 1456

[7] P. H. Coleman, L. Pietronero, Phys. Rep. 213 (1992) 311

[8] J. Kempa, Journ. Physics G 20 (1994) 215

[9] H. H. Mielke et al., Nucl. Instrum. and Meth. A 360 (1995) 367

J. R. Hörandel, FZKA-Report 6015, Forschungszentrum Karlsruhe (1998)

[10] F. Herm et al., Roman. Journ. Physics 38 (1993) 475

[11] H. Rebel, G. Völker, M. Föller, A. A. Chilingarian, Journ. Physics G 21 (1995) 451

[12] J. N. Capdevielle et al., KfK-Report 4998, Kernforschungszentrum Karlsruhe (1990)

D. Heck, J. Knapp, J. N. Capdevielle, G. Schatz, T. Thouw, FZKA-Report 6019, Forschungszentrum Karlsruhe (1998)

[13] H. Fesefeldt, Report PITHA 85/02, RWTH Aachen, Germany (1985)

[14] K. Werner, Phys. Rep. 232 (1993) 87

[15] N. N. Kalmykov and S. S. Ostapchenko, Yad.Fiz. 56 (1993) 105

[16] W. R. Nelson, H. Hiroyama, D. W. O. Rogers, SLAC Report 265 (1985) 
[17] GEANT: CERN Program Library Long Writeups W5013 (1993)

[18] B. B. Mandelbrot, Physica A 163 (1990) 306

[19] A. Haungs, FZKA-Report 5845, Forschungszentrum Karlsruhe (1996)

[20] A. Aharony, Physica A 168 (1990) 479

[21] L. Lönnblad, C. Peterson, T. Rögnvaldsson, CERN Preprint, CERNTH.7135/94 (1994)

[22] H. O. Klages et al. - KASCADE Collaboration, Highlight Talk at the 25th ICRC, Durban, South Africa, 1997, to be published

[23] A. A. Watson, Rapporteur Talk at the 25th ICRC, Durban, South Africa, 1997, to be published

J. Knapp, Rapporteur Talk at the 25th ICRC, Durban, South Africa, 1997, to be published

[24] A. P. Szabo, R. J. Protheroe, Astroparticle Physics 2 (1994) 375

[25] C. E. Fichtel, J. Linsley, Astrophys. Journal 300 (1986) 474

[26] T. Stanev, P. L. Biermann, T. K. Gaisser, Astron. Astrophys. 274 (1993) 902 